Implementing a new procedure for screening and treatment of malnutrition in community care for older people - lessons learnt

a Jane Murphy, ${ }^{a, e}$ Michael Bracher, ${ }^{a}$ Daria Tkacz, ${ }^{c}$ Annemarie Aburrow, ${ }^{b}$ Grace Allmark, ${ }^{b}$ Kathy Steward, 'Kathy Wallis, ${ }^{d}$ Carl May

${ }^{a}$ Faculty of Health and Social Sciences, Bournemouth University, Bournemouth, UK, ${ }^{b}$ Southern Health NHS Foundation Trust, Southampton, UK

${ }^{c}$ Wessex Academic Health Science Network, Chilworth, UK

${ }^{d}$ Faculty of Public Health and Policy, London School of Hygiene and Tropical Medicine, London, UK

${ }^{e}$ School of Health Sciences, University of Southampton, Southampton, UK

Contact: jmurphy@bournemouth.ac.uk

\title{
Accepted for Publication
}

British Journal of Community Nursing April 2020 Vol 25, No 4 


\section{Abstract}

This article reports the implementation of a new procedure for screening and treatment of malnutrition in a community NHS Trust in England. The barriers and facilitators to implementation were assessed with staff from Integrated Community and Older People's Mental Health teams. Data from interviews and surveys were collected at baseline, 2 months after initial training and 16 months after initial training and following deployment of a Nutrition Lead to embed new developments for nutritional care. The adoption of the procedure made screening and treatment of malnutrition simpler and more likely to be actioned. The benefit of a Nutrition Lead and local nutrition champions to support and empower staff (rather than reliance on training alone) was shown to drive change for nutritional care across the community. Prioritisation and commitment of leadership at organisational level is needed to embed and sustain malnutrition screening and treatment in routine practice.

\section{Key words}

Older people, Malnutrition, Screening, Community, Dietitian 
Key points

- Malnutrition is common in older people living in the community

- Older people should be screened to manage and treat malnutrition and prevent the decline nutritional status

- Adoption of this new procedure to provide nutritional care made screening and treatment simpler and actionable

- A Nutrition Lead and local nutrition champions can support and empower staff and provide access to specialist advice for high risk patients

- Strong leadership and governance processes are required to prioritise nutritional care and embed into routine practice

Reflective questions

- Do you have access to training for screening and treatment of malnutrition?

- Are there routine screening measures in place for screening and treatment of malnutrition at your organisation?

- Is nutritional care considered a priority as part of your role?

- Do you have patient information resources to manage malnutrition?

- Do you have access to specialist support and advice on treatment for malnutrition? 


\section{Background}

In the UK, it is estimated that approximately 3 million people are at risk of malnutrition (as undernutrition) of which nearly half are 65 years or over, the majority (93\%) residing in the community (Elia and Russell, 2009). Malnutrition and unintentional weight loss are costly (Elia et al, 2015) and have a significant impact on quality of life due to increased susceptibility to disease and mortality (Soderstrom et al, 2017). Moreover malnutrition poses strain on health care resources due to delayed recovery from illness, increased need for health care provision at home, more frequent visits by nurses and a greater number of hospital admissions (Guest et al, 2011). Malnutrition is largely preventable through appropriate screening using a validated tool such as the Malnutrition Universal Screening Tool ('MUST') (Elia, 2003). National policy guidance (National Institute for Health and Care Excellence (NICE), 2006; 2012) recommends screening for malnutrition leading to substantial cost savings from early identification and treatment largely from reducing healthcare resources (Edington et al, 2004). Screening should be undertaken by trained care providers and integrated into existing pathways of care. After screening, appropriate action should be taken to manage those patients identified to be malnourished or at risk of malnutrition. Malnutrition in the community is optimally treated by providing first-line dietary advice - 'food-first' approach and then in combination with oral nutritional supplements when necessary (Dera and Woodham, 2016; Holdeway et al, 2017).

This article reports the barriers and facilitators to implementation of a new procedure for nutrition screening and care for older people in a selected business unit within Southern Health Foundation Trust (SHFT) and lessons learnt for roll-out and spread. The procedure was informed by Wessex Academic Science Network's (AHSN) Older People's Essential Nutrition (OPEN) Toolkit (Wessex AHSN, 2018). The new procedure outlined the process of care for nursing teams as well as the nutritional advice and support that should be given to older people in accordance with their malnutrition risk, and was developed to reflect local services. Existing policy established when a patient should be screened for malnutrition and the care that a patient should receive depending on their malnutrition risk (assessed by 'MUST' screening). The new procedure introduced a number of updates to better reflect NICE guidelines (NICE, 2006).

They were as follows: 1 ) only patients at medium or high risk of malnutrition (those with a 'MUST' score of 1 or more) who require monthly re-screening and follow-up (previously all patients were expected to receive monthly screening); patients at low risk were to be rescreened annually, unless there is a significant change in their health status, 2) provision of malnutrition information resources sheets to medium and high-risk patients (previously these were not routinely provided to patients in these categories). New information resources were produced as part of the project, and guidance for when to use existing resources were specified within the new procedure, 3) the electronic patient records system $(\mathrm{RiO})$ included a facility for including screening for malnutrition that captured all information from the 'MUST' assessment (previously only the resulting 'MUST' score was recorded). As part of the new procedure, a form was generated within the RiO system to record screening results, to enable care planning activity, 4) the new procedure emphasised that staff at all roles and grades who provide community care to older people have responsibility for malnutrition screening and delivery of appropriate treatment (Bracher et al, 2019a).

At the same time, a research project was undertaken by Bournemouth University (funded by the Burdett Trust for Nursing) - Implementing Nutrition Screening in Community Care for Older People (INSCCOPe). The aim of the project was to explore the factors that may help 
or hinder implementation of the new procedure and longer term embedding of this as a routine aspect of community care (using Normalisation Process Theory; May and Finch, 2009).

Details of the protocol are published elsewhere (Bracher et al, 2019a). In brief, implementation was assessed through observation of staff from Integrated Community or Older People's Mental Health (OPMH) teams. Data collection occurred at three time points: baseline (T0), 2 months following completion of initial training (T1), and 16 months following the initial training and following deployment of a Nutrition Lead as part of embedding new developments in routine nutritional care (T2). Each observation point consisted of a 23-item questionnaire and follow-up semi-structured telephone interviews with staff. Bespoke training via a 1-hour session delivered by a dietitian was provided during the introduction of the new procedure to address the gaps in knowledge, with an emphasis on identifying the underlying causes of malnutrition and establishing a plan of care.

Findings after delivering training (T1)

The results after training at $\mathrm{T} 1$ showed high levels of non-completion of the training and vulnerability to attrition of trained staff. Community-based nurses felt they had some knowledge to help care for patients at risk of malnutrition, but they lacked the specialist support due to the absence of a community dietetic service in their geographical area. There was also lack of monitoring of post-intervention compliance and oversight of the process to assess and treat malnutrition at a higher organisational level, because staff failed to make treating malnutrition a priority (Bracher et al, 2019b). Updates on compliance, training and resource should be provided to senior managers at regular intervals. As such, we suggested a process that links front-line monitoring and resource allocation directly to those with responsibility to ensure successful implementation of the new procedure, thus creating an 'organisational feedback loop'.

The findings demonstrated what aspects of implementation were required to embed new developments in routine nutritional care for older people in community settings. Importantly it informed the deployment a Nutrition Lead to provide training and specialist support, as well as 'Nutrition Link' champion roles to help address these issues on a daily basis within teams. A new Nutrition Lead post for the project was created (Table 1).

The main role of the Nutrition Lead was to provide training to staff on malnutrition, 'MUST', the newly developed malnutrition care pathway within the Trust and appropriate treatment. The face-to-face training was offered to every community nursing and therapy team within the selected business unit and was requested by all teams. The delivery of the training was extended to reach local enhanced recovery nursing teams and respiratory nursing teams during the six-month intervention.

Findings after deployment of Nutrition Lead (T2)

Data collected from surveys and interviews indicated that where the procedure had been adopted it was found to make the screening and treatment of malnutrition more straightforward and more actionable (Murphy et al, 2020). An electronic form supported 
improvements in recording and accessing information as well as guiding the assessment. The training on malnutrition and the 'MUST' screening tool had a positive impact on staff confidence and their understanding of the importance of nutrition, particularly in the context of its influence on other aspects of the welfare of patients. The new procedure was also perceived to make nutrition screening and treatment work less complicated. This finding was reflected in service improvement data collected, showing a 4-5 fold increase in the number of screens completed over the period of the project. Thus, patients who would otherwise been unable to access specialist dietetic support, had benefitted the most from the project. Key people identified were nutrition link nurses to attend study days and feedback to the teams, monitor screening rates and offer support, thus having a positive impact on the team's engagement. For role to have lasting impact, it was suggested that they should receive regular training updates and support from more senior staff. However, concerns were also raised about maintaining the existing knowledge levels among new staff, particularly with high staff turnover levels and the need to make nutrition training mandatory.

The Nutrition Lead was perceived as extremely helpful for both supporting staff and providing specialised support to high risk patients and who had an important impact on bringing the important of nutrition to the forefront. As such, a lack of access to community dietitians was a major gap identified in the ability of staff to provide appropriate treatment. Notably, whilst the procedure generated enthusiasm and brought the issue of nutrition to the forefront amongst competing demands, a strategic directive from senior management within the Trust was identified as a further step necessary to embed the procedure as part of usual practice.

\section{Conclusion}

The project has shown that the delivery of nutritional care was considered less of a priority in the community despite being perceived as important by many staff. It is recognised that nursing and therapy staff already have extremely busy caseloads, so are struggling to complete full and comprehensive nutritional assessments of patients. The introduction of a Nutrition Lead (together with localised nutrition link champions) offers significant benefit to i) drive the agenda for nutrition, ii) provide support and guidance to help staff deliver quality improvements in nutritional care in the community and, iii) offer specialist advise to high risk patients. Where the procedure had been adopted, it led to improvements in the screening and treatment of malnutrition. Education and learning for malnutrition screening should be 'essential' for all staff as part of their role. However, for the adoption of the procedure to be sustained, prioritisation by the senior leadership team and organisational support is vital. New training tools (workbook and film) were developed through the project and are freely downloadable (https://www.bournemouth.ac.uk/research/centres-institutes/ageing-dementiaresearch-centre/implementing-nutrition-screening-community-care-older-people-trainingresources). 
Funding: The project is funded by The Burdett Trust for Nursing.

Conflicts of interests: None declared.

Ethics approval: Ethical approval for the study has been granted through institutional ethical review (Bournemouth University); NHS Heath Research Authority approval (IRAS ID 223214) was granted on 05/04/2017. NHS Research Ethics committee approval was not required for this study, as it involves only staff.

\section{References}

Bracher M, Murphy J, Steward K, Wallis K, May CR. What factors promote or inhibit implementation of a new procedure for screening and treatment of malnutrition in community settings? A prospective process evaluation of the Implementing Nutrition Screening in Community Care for Older People (INSCCOPe) project (UK). BMJ Open. 2019a; 9(2):e023362. https://doi. org/10.1136/bmjopen-2018-023362

Bracher M, Steward K, Wallis K, May CR, Aburrow A, Murphy J. Implementing professional behaviour change in teams under pressure-results from phase one of a prospective process evaluation of a new procedure for screening and treatment of malnutrition in community care for older people (INSCCOPe). BMJ Open. 2019b; 9(8):e025966. https://doi.org/10.1136/bmjopen-2018-025966

Dera M, Woodham D. Treating malnutrition in the community. $\mathrm{Br} \mathrm{J}$ Community Nurs. 2016; 21(11):558-562. https://doi.org/10.12968/bicn.2016.21.11.558

Elia M. The MUST Report. Nutritional screening of adults: a multidisciplinary responsibility. 2003. https://tinyurl.com/ubwtr8l (accessed 10 February 2020)

Elia M, Russell CA. Combating malnutrition: recommendations for action. 2009. https://tinyurl.com/btqysd (accessed 10 February 2020)

Elia M. The cost of Malnutrition in England and potential cost savings from nutritional interventions. 2015. https://tinyurl.com/hggbfga (accessed 10 February 2020)

Guest JF, Panca M, Baeyens JP et al. Health economic impact of managing patients following a community-based diagnosis of malnutrition in the UK. Clin Nutr. 2011; 30(4):422-429. https://doi.org/10.1016/..clnu.2011.02.002

Holdoway A, Anderson L, McGregor I et al. A guide to managing adult malnutrition in the community. 2nd edn. 2017. https://tinyurl.com/y7kj8soy (accessed 10 February 2020)

Murphy JL, Tkacz D, Bracher M et al. Implementing nutrition screening in the communityresults from phase two a prospective process evaluation of a new procedure for screening and treatment of malnutrition in community care for older people (INSCCOPe-phase two). Clinical Nutrition ESPEN. 2020 (in press)

National Institute for Health and Care Excellence. Nutrition support for adults: oral nutrition support, enteral tube feeding and parenteral nutrition. CG32. https:// tinyurl.com/ppy6w8t (accessed 10 February 2020) 
National Institute for Health and Care Excellence. Nutrition support in adults. 2012. https://tinyurl.com/r2j42qe (accessed 10 February 2020)

Soderstrom L, Rosenblad A, Thors Adolfsson E, Bergkvist L. Malnutrition is associated with increased mortality in older adults regardless of the cause of death. $\mathrm{Br} \mathrm{J}$ Nutr. 2017; 117(4):532-540. https://doi.org/10.1017/S0007114517000435

Wessex Academic Health Science Network. Undernutrition awareness and training. 2018. https://tinyurl.com/qronp6r (accessed 10 February 2020) 


\section{Table1. Aims of the Nutrition Lead role}

- To develop a Nutrition Link role to be implemented in all community nursing teams

- To work in clinical teams to raise the profile of assessing and treating undernutrition and increasing specialist support available

- To train registered and non-registered staff on the new procedure, including screening using the Malnutrition Universal Screening Tool ('MUST') and how to implement appropriate care plans and follow up

- To raise the profile of the importance of nutritional assessment through the trust wide Nutrition and Hydration Committee

- To develop training and support materials to build in sustainability.

- To support the roll out of the new procedure across community services 